\title{
The VMC Survey - XXXV. Model fitting of LMC Cepheid light curves
}

\author{
Fabio Ragosta, ${ }^{1,2,3}$ Marcella Marconi, ${ }^{3}$ Roberto Molinaro, ${ }^{3 \star}$ Vincenzo Ripepi, ${ }^{3}$ \\ Maria Rosa L. Cioni, ${ }^{4}$ Maria Ida Moretti, ${ }^{3}$ Martin A.T. Groenewegen, ${ }^{5}$ \\ Samyaday Choudhury, ${ }^{6,7}$ Richard de Grijs, ${ }^{6,7,8}$ Jacco Th. van Loon, ${ }^{9}$ \\ Joana M. Oliveira, ${ }^{9}$ Valentin D. Ivanov ${ }^{10}$ Carlos Gonzalez-Fernandez ${ }^{11}$ \\ ${ }^{1}$ Dipartimento di Fisica, Via Cinthia, I-80126 Fuorigrotta, Naples, Italy \\ ${ }^{2}$ INFN sez. di Napoli Compl. Univ. di Monte S. Angelo, Edificio G, Via Cinthia, I-80126 - Napoli, Italy \\ ${ }^{3}$ INAF-Osservatorio Astronomico di Capodimonte, via Moiariello 16, I-80131, Naples, Italy \\ ${ }^{4}$ Leibniz-Institut für Astrophysik Potsdam, An der Sternwarte 16, D-14482 Potsdam, Germany \\ ${ }^{5}$ Koninklijke Sterrenwacht van België, Ringlaan 3, B-1180 Brussels, Belgium \\ ${ }^{6}$ Department of Physics and Astronomy, Macquarie University, Balaclava Road, Sydney, NSW 2109, Australia \\ ${ }^{7}$ Research Centre for Astronomy, Astrophysics and Astrophotonics, Macquarie University, Balaclava Road, Sydney, NSW 2109, Australia \\ ${ }^{8}$ International Space Science Institute-Beijing, 1 Nanertiao, Zhongguancun, Hai Dian District, Beijing 100190, PR China \\ ${ }^{9}$ Lennard-Jones Laboratories, School of Chemical and Physical Sciences, Keele University, ST5 5BG, UK \\ ${ }^{10}$ European Southern Observatory, Karl-Schwarzschild-Str. 2, D-85748 Garching bei München, Germany \\ ${ }^{11}$ Institute of Astronomy, University of Cambridge, Madingley Road, Cambridge CB3 OHA, UK
}

Accepted XXX. Received YYY; in original form ZZZ

\begin{abstract}
We present the results of the light curve model fitting technique applied to optical and near-infrared photometric data for a sample of 18 Classical Cepheids (11 fundamentals and 7 first overtones) in the Large Magellanic Cloud (LMC). We use optical photometry from the OGLE III database and near-infrared photometry obtained by the European Southern Observatory public survey "VISTA near-infrared survey of the Magellanic Clouds system". Iso-periodic nonlinear convective model sequences have been computed for each selected Cepheid in order to reproduce the multi-filter light curve amplitudes and shape details. The inferred individual distances provide an intrinsic weighted mean value for the LMC distance modulus of $\mu_{0}=18.56 \mathrm{mag}$ with a standard deviation of $0.13 \mathrm{mag}$. We derive also the Period-Radius, the Period-Luminosity and the Period-Wesenheit relations that are consistent with similar relations in the literature. The intrinsic masses and luminosities of the best-fitting models show that all the investigated pulsators are brighter than the predictions of the canonical evolutionary mass-luminosity relation, suggesting a significant efficiency of non-canonical phenomena, such as overshooting, mass loss and/or rotation.
\end{abstract}

Key words: stars: variables: Cepheids - stars: oscillations - galaxies: Magellanic Clouds - galaxies: structure

\section{INTRODUCTION}

Classical Cepheids (CCs) are a class of pulsating stars widely used to calibrate the extragalactic distance scale, through secondary distance indicators. Their role as tool to measure distance is based on the relation they show between the period of pulsation and their intrinsic luminosity, known as Period-Luminosity (PL) relation. From an evolutionary point of view, a $\mathrm{CC}$ is a star with an intermediate mass

^ E-mail: roberto.molinaro@inaf.it (from $3 \mathrm{M}_{\odot}$ to $13 \mathrm{M}_{\odot}$ ), in the central helium burning phase (covering an age range from $\sim 10 \mathrm{Myr}$ to $\sim 200 \mathrm{Myr}$, see Anderson et al. 2017), that passes through the instability strip as it evolves bluewards and then redwards (blue loop excursion), at roughly constant luminosity for each given mass. From a theoretical point of view, CCs, like all the other classes of pulsating stars, obey to a relation between the oscillation period and their mean density, as demonstrated by Eddington (1926). By combining this relation with the Stefan-Boltzmann law one obtains a Period-LuminosityMass-Temperature relation. For CCs, this relation can be 
reduced to a Period-Luminosity-Color (PLC) relation, because the theory of stellar evolution predicts the existence of a mass-luminosity relation (MLR), whose coefficients depend on the assumed metal and helium abundances. Currently adopted PL relation can be seen as a projection of the PLC relation on the PL plane (Freedman et al. 1991; Bono, Caputo, Castellani \& Marconi 1999; Caputo et al. 2005 , and references therein). In other words, the PL relation shows an intrinsic dispersion related to the finite width of the instability strip. Obviously, for each filter combination, both the PLC and its projection on the PL plane critically depend on the MLR. Since the efficiency of noncanonical phenomena (such as core overshooting, mass loss, rotation) significantly affects the MLR, in order to determine precise distances using CCs, a detailed theoretical evaluation of the impact of these processes needs to be assessed.

Several authors have discussed the effect of mass loss and core overshooting (see Chiosi et al. 1993; Bono, Caputo, Castellani \& Marconi 1999; Caputo et al. 2005; Keller et al. 2006; Neilson et al. 2012; Marconi et al. 2013, and references therein), as well as of rotation (Anderson et al. 2016) on CC properties. These studies are often related to the so called mass discrepancy problem, first outlined by Stobie et al. (1969) and Christy (1970) and subsequently confirmed by additional investigations. According to these studies, the $\mathrm{CC}$ evolutionary mass (inferred from the comparison between theoretical isochrones and observations in the color-magnitude diagram) was found to be systematically higher than the "pulsational" one based on the Period-Mass-Radius relation ${ }^{1}$ (Fricke et al. 1972; Bono et al. 2001) or other methods based on the theory of pulsation (Bono et al. 2002; Caputo et al. 2005). Keller et al. (2006) and Marconi et al. (2013) adopted the model fitting of multi-filter light, radial velocity and radius curves to address the mass discrepancy. This is done through direct comparison of the observed and predicted variations along a pulsation cycle, the latter based on nonlinear convective pulsational models (see Bono et al. 2000, 2002; Marconi et al. 2013, for a detailed discussion of the method).

The "VISTA near-infrared $Y, J, K_{\mathrm{s}}$ survey of the Magellanic Clouds system" (VMC - Cioni et al. 2011) covers the Magellanic system with deep Near-Infrared (NIR) ( $Y, J, K_{\mathrm{s}}$ filters) VIRCAM (VISTA InfraRed Camera; Dalton et al. 2006) photometry using the ESO/VISTA telescope (Emerson et al. 2006). The main science goals of the VMC are the study of the spatially-resolved star-formation history $(\mathrm{SFH})$ and the determination of the 3D structure of the whole Magellanic system. Particularly useful for the latter aim are pulsating variables such as RR Lyrae stars and CCs that have been the subject of several studies in the context of the VMC survey, as distance indicators and stel-

1 From the combination of the Period-density relation and the Stefan-Boltzman law it is also possible to obtain a PeriodMass-Radius relation, which is useful to estimate the masses of Cepheids if their radii are known, and vice versa. According to the linear adiabatic theory the pulsation period of variables is related to mass and radius through the equation $P=\alpha\left(M / M_{\odot}\right)^{\beta}\left(M / M_{\odot}\right)^{\gamma}$ (Fricke et al. 1972), which can be linearized easily in logarithmic space $(\log P=\log \alpha+$ $\left.\beta \log \left(M / M_{\odot}\right)+\gamma \log \left(R / R_{\odot}\right)\right)$ thus obtaining the PMR relation. lar population tracers (see e.g. Ripepi et al. 2012a,b, 2014, 2015, 2016, 2017; Moretti et al. 2014, 2016; Muraveva et al 2015, 2018).

Marconi et al. (2017) presented the model fitting of multi-wavelength light curves and, when available, radial velocity curves of 12 Small Magellanic Cloud (SMC) CCs whose NIR observations were secured as part of the VMC data. The inferred stellar parameters and individual distances permitted to constrain not only the mean distance modulus of the SMC but also the behaviour of the investigated stars in the MLR, PL, Period-Radius (PR) and Period-Wesenheit (PW) relations. ${ }^{2}$

In this paper we extend this to a sample of 11 fundamental (F) and 7 first overtone (FO) CCs in the Large Magellanic Cloud (LMC), that are within the field of view of the VMC survey.

As regards the organization of the paper, in Section 2 we discuss the sample selection, in Section 3 we describe the adopted model fitting technique. The application of this technique to the selected LMC CCs and the implications of our results for the MLR, the PR, the PL and PW relations are described in sections 4 and 5 respetively. The final section includes a summary and perspectives.

\section{SELECTION OF THE SAMPLE}

The selected sample of CCs is composed of 11 and $7 \mathrm{~F}$ and FO pulsators, respectively, that cover a range in oscillation period from $\sim 1$ to $\sim 30$ days. The selected CCs have optical photometry from the OGLE III database ${ }^{3}$ (Soszyński et al. 2010) and NIR photometry from the VMC database (see e.g. Cioni et al. 2011; Ripepi et al. 2016, 2017, for a description of VMC light curves). In particular we used aperture photometry data from the Cambridge Astronomy Survey Unit (CASU) and the Vista Science Archive (VSA)(see Cross et al. 2012; González-Fernández et al. 2018, for details). The sample is selected in order to span a wide range in period, luminosity and shape of the light curves from the OGLE III database. The period values spanned by our sample do not include the range of the so called 'bump' Cepheids (8-12 days). However, this period range has already been analyzed, using the same pulsating code as in the current work, in Bono et al. (2002), where the authors selected a couple of LMC Cepheids, one with the bump on the rising branch and the other on the decreasing branch.

Altough the number of the selected CCs does not represent the entire LMC, they let us test the prediction capabilities of the model fitting technique in a stellar system. We

2 The Wesenheit magnitudes include a color term with a coefficient that corresponds to the ratio between total to selective extinction in the selected filter pair (Madore et al. 1982; Caputo, Marconi \& Musella 2000), thus making the Wesenheit relations reddening free.

3 When this work began OGLE IV data were not available. We checked for possible changes between the two data releases finding that the number of points in the $V$ and $I$ bands does not increase by more than a few percents for most sources in our sample. Only for three stars the photometric observations increase significantly, but this does not affect the results of our method, because a good phase coverage of light curves was already available in OGLE III. 


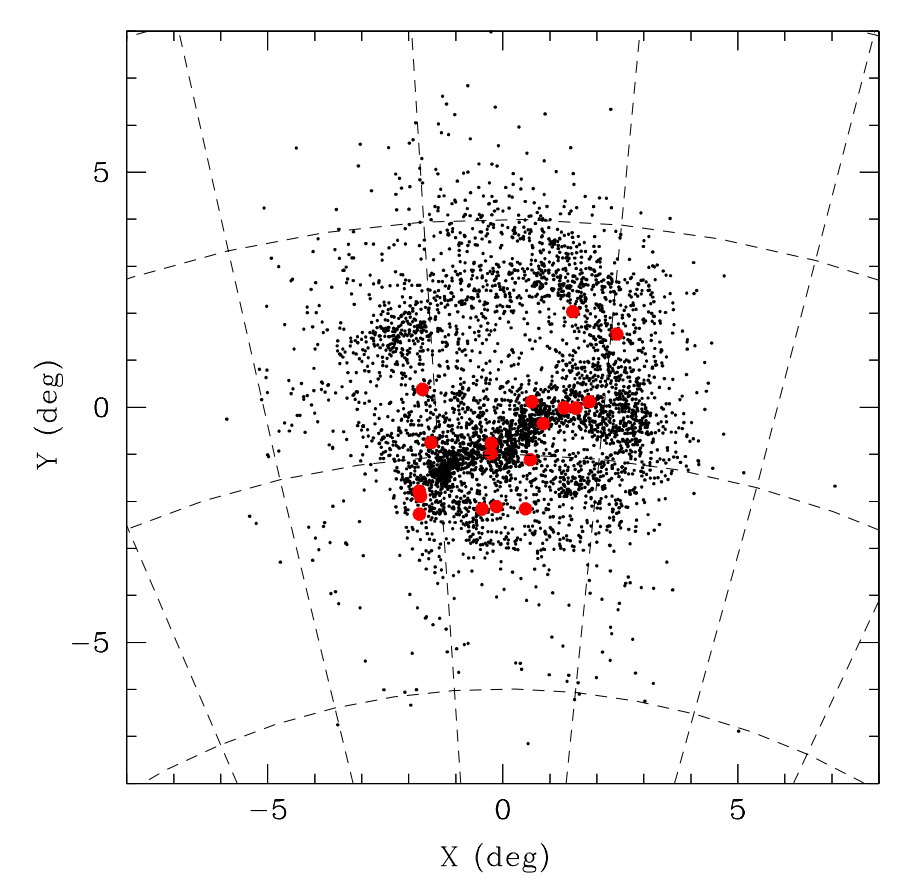

Figure 1. Distribution on the sky of the CCs investigated in this paper (red filled circles). For comparison the whole sample of known CCs in the LMC from the OGLE survey (black dots) is shown. X and Y are defined as in van der Marel \& Cioni (2001) with $\alpha_{0}=81^{\circ}$ and $\delta_{0}=-69^{\circ}$

note that obtaining a statistically significant extension of the selected target number would be extremely time consuming to reach convergence of our hydrodynamical pulsation code.

The distribution in right ascension and declination of the selected CCs is shown in Fig.1, where all known CCs in the LMC from the OGLE survey (Soszyński et al. 2015) are shown for comparison. The identification, the period, and the mean visual magnitude, of the selected CCs are listed in the first three columns of Table 1; these values were taken from Soszyński et al. (2015). As shown in Table 1 the selected CC sample encompasses wide ranges of periods and mean magnitude thus allowing us to check the predictive capabilities of the model fitting technique over a large interval of CC observed properties.

\section{THE MODEL FITTING TECHNIQUE}

The fitting technique adopted to find the best model reproducing the observations, is similar to that described in Marconi et al. (2017):

- both observed and modeled photometric curves are phased in order to have the maximum of light at the same phase in a given reference band: e.g. in this work the maximum of light in the $V$ band is at phase 0 .

- for each modeled photometric band, we estimated the shifts in magnitude $(\delta \mu)$ and phase $(\delta \phi)$ that provide the best match between modeled and observed light curves.
Specifically, these two parameters have been obtained by minimizing the following $\chi^{2}$ equation:

$\chi^{2}=\frac{1}{N_{\mathrm{bands}}} \sum_{j}^{\mathrm{N}_{\mathrm{bands}}} \frac{1}{N_{\mathrm{DOF}}^{j}} \sum_{i}^{\mathrm{N}_{\mathrm{pts}}^{j}} \frac{\left[\mathrm{m}_{i}^{j}-\left(M_{\mathrm{mod}}^{j}\left(\phi_{i}^{j}+\delta \phi^{j}\right)+\delta \mu^{j}\right)\right]^{2}}{\sigma_{i}^{j}}$

where the two indices $j$ and $i$ run respectively on the number of bands, $N_{\text {bands, }}$, and on the number of epochs, $N_{\text {pts }}$, the observed phases, magnitudes and errors are indicated with $\phi_{i}^{j}$ and $\mathrm{m}_{i}^{j}, \sigma_{i}^{j}$ respectively, while $M^{j}$ is the absolute magnitude of the pulsating model evaluated at the same phase of observations plus the shift $\delta \phi$. To evaluate the model at a given phase the theoretical light curves have been interpolated using a smooth spline. In the above formula, the term $N_{D O F}^{j}=N_{\mathrm{pts}}^{j}-2$ is the number of degrees of freedom for the $\mathrm{j}$-th band. We note that, because the initial phasing procedure, described above, is the same for observations and models, the fitted values of $\delta \phi$ are typically small $(\sim 0.02)$ and represent just fine tuning phase shift values. On the other hand, the fitted parameter $\delta \mu^{j}$ represents the apparent distance modulus in the $j^{\text {th }}$ band.

Following the same approach as in Marconi et al. (2017), for each selected target CC, we built isoperiodic model sequences at fixed mass, varying the effective temperature and in turn the luminosity level. This allowed us to obtain a sample of light curve models with different shapes, magnitudes and periods. Using this sample of models, we selected the model best matching the observed curves. The isoperiodic sequences are built using the typical elemental composition of the LMC ( $Y=0.25 ; Z=0.008$; see Romaniello et al. 2008; Luck et al. 1998, for details). Once we found the best fitting $T_{\mathrm{e}}$, we built another sample of models varying the mass but fixing the obtained best fitting temperature, again selecting the best fitting model matching the observed curves. Thus we were able to evaluate the mass, the luminosity, the effective temperature of the star and in turn its individual apparent distance modulus in each selected band. 

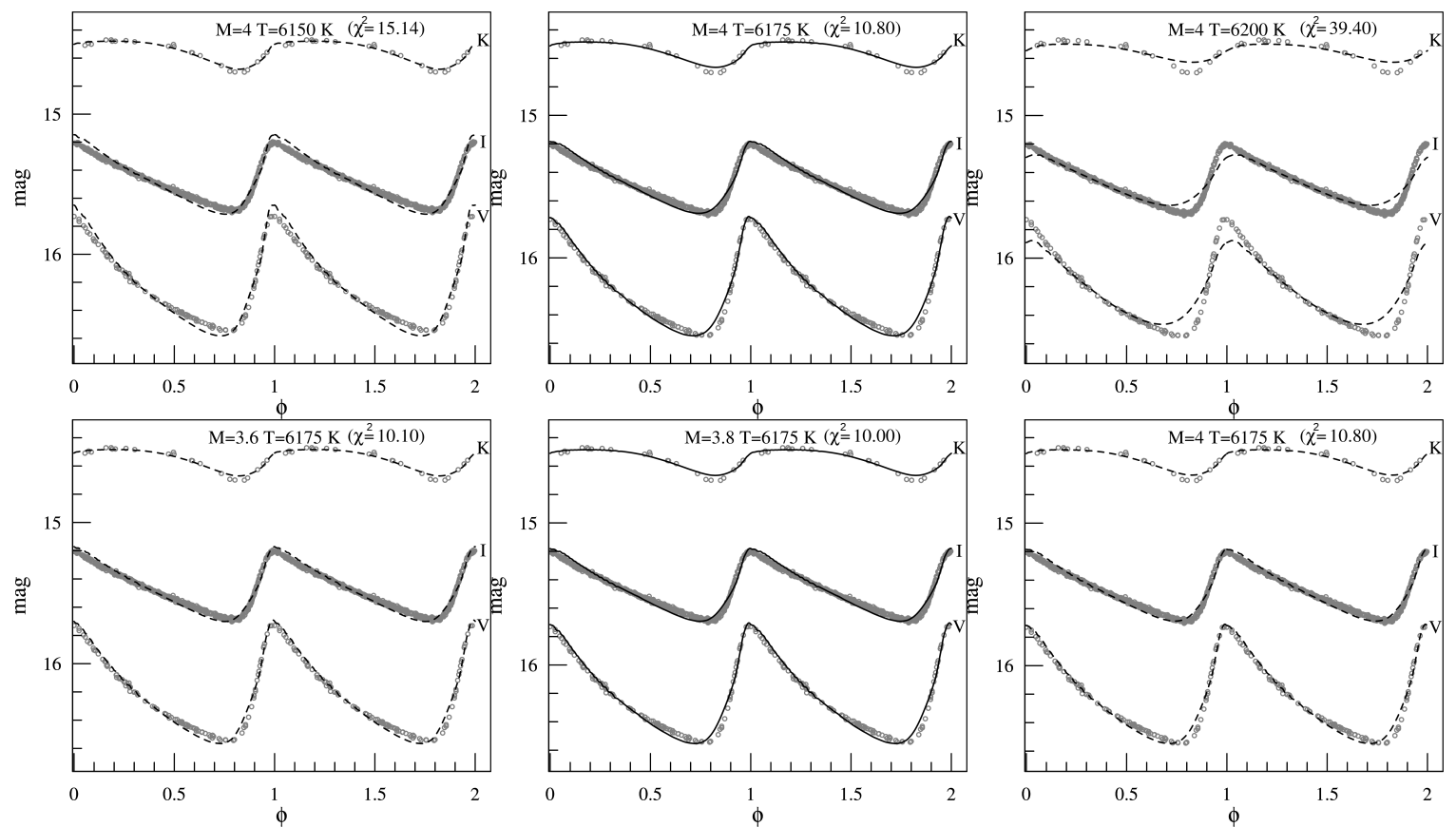

Figure 2. Comparison of the observed $V, I$ and $K_{s}$ light curves of the variable OGLE LMC CEP 2138 and the fitted theoretical light curves. Data are plotted with grey symbols, while models are plotted with lines. In the top panels we show the models calculated assuming a fixed mass, namely $\left(M=4.0 \mathrm{M}_{\odot}\right)$ and varying effective temperature. Once the model with best $T_{e}$ (namely $\left.T_{e}=6175 \mathrm{~K}\right)$ is found, (solid line), the effective temperature is maintained fixed at its best value and the $\chi^{2}$ in equation 1 is minimized by varying the mass. Models with varying mass and fixed $T_{e}$ are showed in the bottom panels, where the final best fitting model is again indicated with a solid line and is characterized by $T_{e}=6175 \mathrm{~K}$ and $M=3.8 \mathrm{M}_{\odot}$. The $\chi^{2}$ values of the fit are also labeled in each panel. 

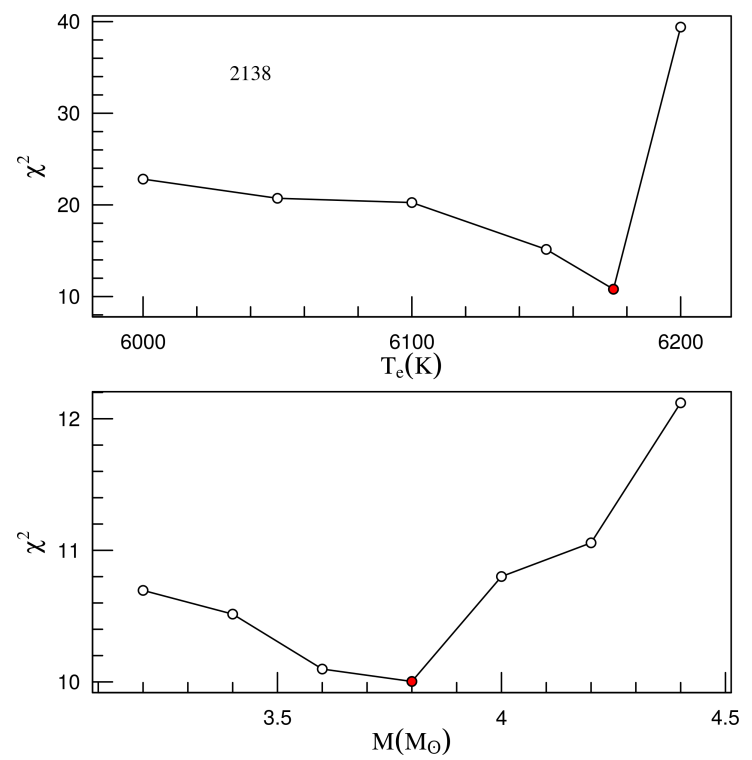

Figure 3. Total reduced $\chi^{2}$ values obtained from the fitting procedure applied to $O G L E \_L M C \_C E P \_2138$ are shown as a function of the model effective temperature (top) and mass (bottom). The values of the best effective temperature and mass are indicated by the red dots.

In Fig. 2 we show an example of the model fitting dependence on the assumed effective temperature (fixed mass $M=4.0 \mathrm{M}_{\odot}$ ) and stellar mass (fixed $T_{\text {eff }}=6175 \mathrm{~K}$ ). The $\chi^{2}$ analysis for models with fixed mass identifies as best fit effective temperature $T_{\text {eff }}=6175 \mathrm{~K}$, as shown in the top panel of the quoted figure. Then varying the mass (Fig. 2 bottom panels), one obtains the best fitting value $M=3.8 \mathrm{M}_{\odot}$. Since it is difficult to evaluate by-eye the quality of the fit shown in Fig. 2, in Fig. 3 we show the total reduced $\chi^{2}$ as a function of the model effective temperature (top) and mass (bottom)

Looking at the best $\chi^{2}$ value in the figure and also at those reported in the Tab. 1 it is evident that they are not always close to the expected canonical value $\chi^{2}=1$. This is due both to error underestimation of the observations and to the difficulty to reproduce exactly light curves with more complex shapes (see also Fig. 5). The presence of features in the light curves of pulsating variables, which make them more complex from the shape point of view, is due to the coupling between pulsation and convection which becomes more important moving towards the red boundary of the instability strip (see Bono, Marconi \& Stellingwerf 1999, and references therein). On this basis, we expect a correlation between the $\chi^{2}$ values and the best fitting effective temperature in the sense that lower $\chi^{2}$ values correspond to higher effective temperatures. This trend is evident in Fig. 4 where the $\chi^{2}$ values of Table 1 are plotted against the best fitting effective temperature $T_{e}{ }^{4}$, with lager $\chi^{2}$ values populating the zone of lower effective temperaures. Moreover a clear separation can be seen between F and FO models, the latter having smaller $\chi^{2}$ values and as well as known higher effective temperatures.

${ }^{4}$ Note that the source OGLE_LMC_CEP_0546 does not appear in Fig. 4 because of its $\chi^{2}$ value is out of the y-axis range.

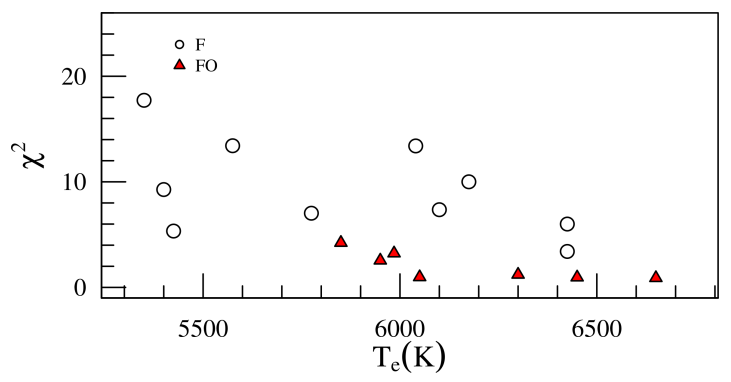

Figure 4. The $\chi^{2}$ values obtained from the fitting procedure and listed in Table 1 are plotted against the best values of effective temperature for all sources of the selected sample: $\mathrm{F}$ pulsators are shown using empty circles, while FOs are plotted with red triangles. 
Table 1. Properties of the target CCs and of the associated best-fitting models. From left to right: OGLE identification, observed period, mean $V$ magnitude and pulsation mode (from the OGLE III database Soszyński et al. 2010), best-fitting model mass, luminosity, effective temperature, mixing length parameter, inferred distance modulus in the $V, I$, and $K_{\mathrm{s}}$ bands and associated errors, absolute distance modulus with associated error, magnitude correction to refer distance modulus to LMC barycenter, reddening with associated error, mean radius and $\chi^{2}$ value. For all CCs the error in the inferred mass and temperature is $\Delta T=25 \mathrm{~K}$ and $\Delta M=0.2 \mathrm{M}_{\odot}$, respectively; the assumed composition is $Y=0.25, Z=0.008$, except for $O G L E \_L M C \_C E P \_2019$ for which $Y=0.30$ (see text).

\begin{tabular}{|c|c|c|c|c|c|c|c|c|c|c|c|c|c|c|c|}
\hline ID & $\begin{array}{c}\mathrm{P} \\
\text { (days) }\end{array}$ & $\begin{array}{c}\langle V\rangle \\
(\mathrm{mag})\end{array}$ & Mode & $\begin{array}{c}M \\
\left(\mathrm{M}_{\odot}\right)\end{array}$ & $\begin{array}{c}\log \left(L / L_{\odot}\right) \\
\quad(\operatorname{dex})\end{array}$ & $\begin{array}{l}T_{e f f} \\
(\mathrm{~K})\end{array}$ & $\alpha_{M L}$ & $\begin{array}{c}\mu_{V} \\
\text { (mag) }\end{array}$ & $\begin{array}{c}\mu_{I} \\
(\mathrm{mag})\end{array}$ & $\begin{array}{c}\mu_{K} \\
(\mathrm{mag})\end{array}$ & $\begin{array}{c}\mu_{0} \\
(\mathrm{mag})\end{array}$ & $\begin{array}{l}\mu_{0}^{\text {corr }} \\
\text { (mag) }\end{array}$ & $\begin{array}{c}E(B-V) \\
\quad(\mathrm{mag})\end{array}$ & $\begin{array}{c}R \\
\left(\mathrm{R}_{\odot}\right)\end{array}$ & $\chi^{2}$ \\
\hline 1481 & 922 & 17.28 & FO & 3.00 & 2.62 & 6650 & 1.50 & $19.14 \pm 0.10$ & $18.89 \pm 0.07$ & $18.49 \pm 0.08$ & $18.42 \pm 0.09$ & 0.002 & $0.24 \pm 0.05$ & 15.4 & 0.9 \\
\hline 3131 & 1.095 & 17.01 & FO & 2.80 & 2.63 & 6450 & 1.80 & $18.89 \pm 0.05$ & $18.72 \pm 0.03$ & $18.52 \pm 0.02$ & $18.47 \pm 0.02$ & 0.02 & $0.14 \pm 0.01$ & 16.6 & 1.0 \\
\hline 3004 & 1.524 & 17.15 & $\mathrm{~F}$ & 3.00 & 2.65 & 6425 & 1.60 & $19.08 \pm 0.08$ & $18.86 \pm 0.04$ & $18.55 \pm 0.01$ & $18.48 \pm 0.01$ & -0.04 & $0.19 \pm 0.03$ & 17.2 & 6.0 \\
\hline 1523 & 1.572 & 16.88 & $\mathrm{~F}$ & 2.80 & 2.64 & 6425 & 1.60 & $18.78 \pm 0.08$ & $18.62 \pm 0.04$ & $18.44 \pm 0.02$ & $18.39 \pm 0.02$ & 0.05 & $0.13 \pm 0.02$ & 17.0 & 3.4 \\
\hline 3113 & 2.068 & 16.08 & FO & 5.20 & 3.15 & 6300 & 1.60 & $19.27 \pm 0.05$ & $19.06 \pm 0.03$ & $18.77 \pm 0.01$ & $18.71 \pm 0.01$ & 0.008 & $0.18 \pm 0.02$ & 31.5 & 1.2 \\
\hline 2138 & 3.011 & 16.21 & $\mathrm{~F}$ & 3.80 & 3.03 & 6175 & 1.70 & $19.04 \pm 0.07$ & $18.85 \pm 0.05$ & $18.60 \pm 0.05$ & $18.54 \pm 0.05$ & 0.04 & $0.16 \pm 0.02$ & 28.7 & 10.0 \\
\hline 3105 & 3.514 & 15.38 & FO & 4.80 & 3.33 & 6050 & 1.60 & $18.96 \pm 0.06$ & $18.82 \pm 0.04$ & $18.62 \pm 0.03$ & $18.58 \pm 0.03$ & 0.011 & $0.13 \pm 0.01$ & 42.2 & 1.0 \\
\hline 0961 & 3.711 & 15.87 & $\mathrm{~F}$ & 3.90 & 3.11 & 6100 & 1.70 & $18.90 \pm 0.07$ & $18.76 \pm 0.06$ & $18.54 \pm 0.07$ & $18.49 \pm 0.07$ & 0.018 & $0.13 \pm 0.02$ & 32.5 & 7.4 \\
\hline 1475 & 4.387 & 15.19 & FO & 5.60 & 3.48 & 5985 & 1.51 & $19.14 \pm 0.09$ & $19.01 \pm 0.08$ & $18.77 \pm 0.06$ & $18.73 \pm 0.06$ & 0.03 & $0.13 \pm 0.01$ & 51.2 & 3.2 \\
\hline 1124 & 4.457 & 15.81 & $\mathrm{~F}$ & 5.00 & 3.29 & 6040 & 1.70 & $19.24 \pm 0.08$ & $19.06 \pm 0.06$ & $18.79 \pm 0.03$ & $18.73 \pm 0.03$ & 0.014 & $0.16 \pm 0.02$ & 40.5 & 13.4 \\
\hline 1310 & 5.126 & 17.28 & FO & 5.70 & 3.54 & 5950 & 1.49 & $18.78 \pm 0.04$ & $18.71 \pm 0.05$ & $18.60 \pm 0.05$ & $18.58 \pm 0.06$ & 0.015 & $0.07 \pm 0.01$ & 55.5 & 2.6 \\
\hline 0813 & 5.914 & 14.54 & $\mathrm{FO}$ & 7.00 & 3.66 & 5850 & 1.53 & $18.93 \pm 0.07$ & $18.87 \pm 0.05$ & $18.75 \pm 0.03$ & $18.73 \pm 0.03$ & 0.02 & $0.07 \pm 0.01$ & 66.2 & 4.2 \\
\hline 2012 & 7.458 & 14.95 & $\mathrm{~F}$ & 6.50 & 3.54 & 5775 & 1.90 & $18.98 \pm 0.03$ & $18.88 \pm 0.02$ & $18.71 \pm 0.01$ & $18.68 \pm 0.01$ & 0.008 & $0.10 \pm 0.01$ & 59.4 & 7.0 \\
\hline 1954 & 12.950 & 14.61 & $\mathrm{~F}$ & 5.30 & 3.69 & 5575 & 1.90 & $19.00 \pm 0.07$ & $18.81 \pm 0.05$ & $18.61 \pm 0.02$ & $18.55 \pm 0.02$ & 0.04 & $0.14 \pm 0.02$ & 75.8 & 13.4 \\
\hline 0546 & 15.215 & 14.03 & $\mathrm{~F}$ & 5.20 & 3.77 & 5575 & 1.70 & $18.59 \pm 0.11$ & $18.58 \pm 0.11$ & $18.57 \pm 0.10$ & $18.56 \pm 0.11$ & -0.003 & $0.01 \pm 0.02$ & 83.9 & 44.2 \\
\hline 1086 & 17.201 & 14.35 & $\mathrm{~F}$ & 5.40 & 3.75 & 5350 & 1.90 & $18.86 \pm 0.09$ & $18.76 \pm 0.08$ & $18.64 \pm 0.08$ & $18.61 \pm 0.08$ & -0.03 & $0.08 \pm 0.02$ & 89.5 & 17.7 \\
\hline 2944 & 20.320 & 14.30 & $\mathrm{~F}$ & 6.90 & 3.95 & 5400 & 1.84 & $19.30 \pm 0.06$ & $19.15 \pm 0.06$ & $18.83 \pm 0.07$ & $18.78 \pm 0.08$ & -0.010 & $0.17 \pm 0.02$ & 108.6 & 9.3 \\
\hline 2019 & 28.103 & 13.64 & $\mathrm{~F}$ & 7.70 & 4.11 & 5425 & 1.70 & $19.00 \pm 0.07$ & $18.83 \pm 0.06$ & $18.67 \pm 0.04$ & $18.62 \pm 0.05$ & 0.013 & $0.12 \pm 0.02$ & 131.9 & 5.3 \\
\hline
\end{tabular}


The VMC Survey - XXXV. Model fitting of LMC Cepheid light curves.
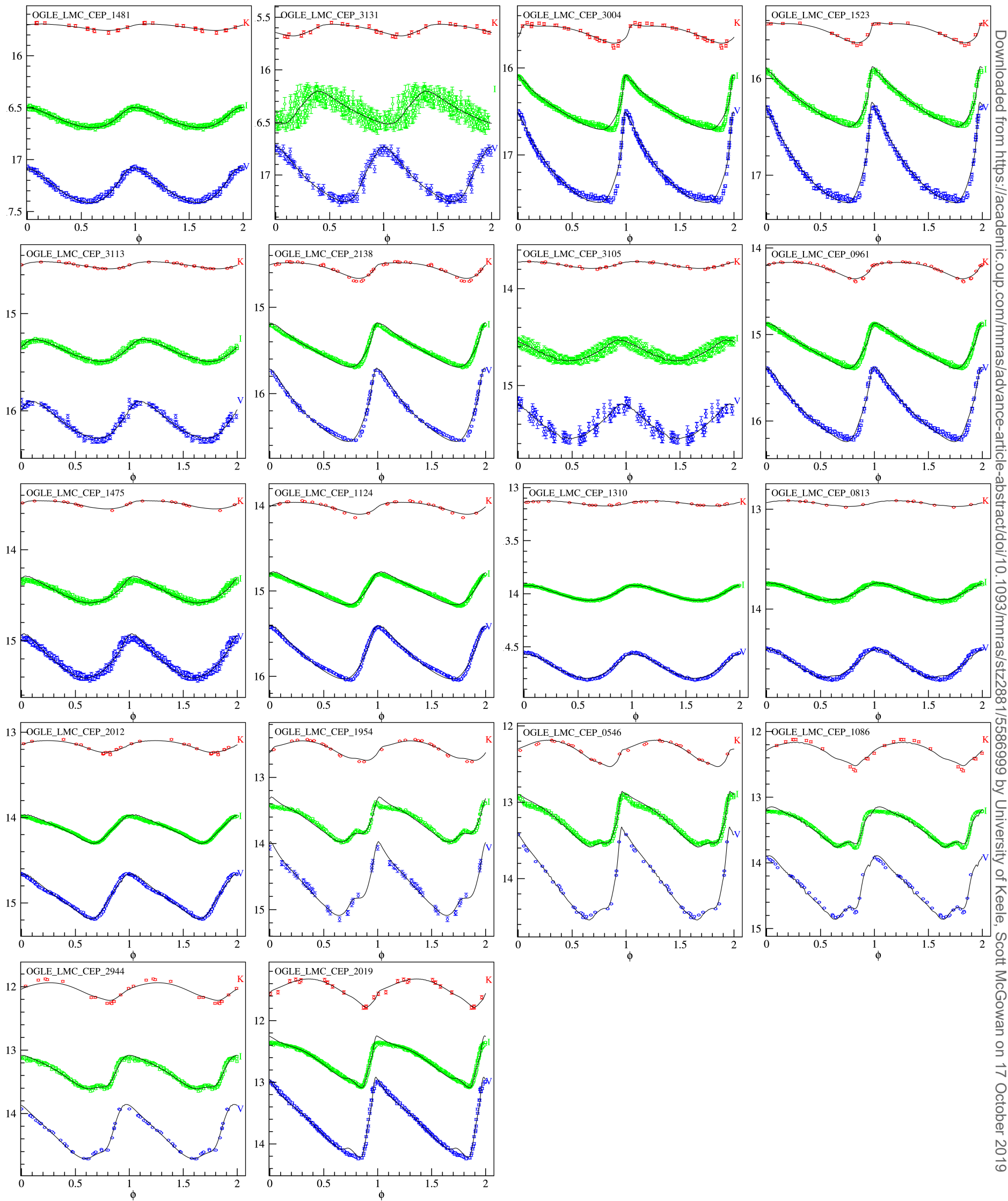

Figure 5. Best fitting models for all selected CCs compared with the observed light curves. Black lines represent the theoretical models, while the $V, I$ and $K_{\mathrm{S}}$ observed light curves are labeled together with star identifications. 


\section{APPLICATION TO THE SELECTED VARIABLES}

The procedure detailed in the previous section was applied to all CCs in Table 1 and the corresponding models are shown in Fig. 5.

We note that for the longest period $\mathrm{CC}$ in our sample (namely $O G L E \_L M C \_C E P \_2019$ ) we had to vary also the elemental composition in order to reproduce the observed light curves. For this star we adopted $Y=0.30, Z=0.008$. The inferred intrinsic stellar parameters, namely the effective temperature, the luminosity and the mass of the best fitting models are reported in Table 1 with their errors.

In particular, the errors on the parameters obtained from the fitting procedure are estimated as the difference between the best values and the parameters of the closest models to the best fitting one on the Mass-Temperature grid. As regard the error on the mass and temperature of the best model, we have adopted the steps of the MassTemperature model grid $\left(0.2 \mathrm{M}_{\odot}\right.$ and $25 \mathrm{~K}$ respectively) generated for our analysis.

The quoted table contains also the unreddened distance moduli $\mu_{0}$ and the $E(B-V)$ values for all stars considered in the present work. They have been calculated by fitting the Cardelli law (Cardelli et al. 1989) to the inferred apparent distance moduli in the $V, I, K_{\mathrm{s}}$ bands.

A simple statistical analysis of the values reported in Tab. 1 gives a mean value of the reddening equal to $E(B-V)=0.13 \mathrm{mag}$ with a standard deviation of $0.05 \mathrm{mag}$, while the inferred mean distance modulus for the LMC is equal to $\mu_{0}=18.59 \mathrm{mag}$ with a standard deviation equal to 0.12 mag. Weighting the fitted parameters with their errors provides almost the same values, with mean distance modulus that is equal to $\mu_{0}^{\mathrm{wt}}=18.63 \mathrm{mag}\left(\sigma^{\mathrm{wt}}=0.10 \mathrm{mag}\right)$ and the mean reddening $E(B-V)^{\mathrm{wt}}=0.11 \mathrm{mag}\left(\sigma^{\mathrm{wt}}=0.04\right.$ mag).

In order to take into account the effect of the inclination of the plane of the LMC with respect to the sky on the barycentric distance estimation, we have also calculated the magnitude corrections (see Table 1) for every $\mathrm{CC}$ of our sample according to the geometric model by van der Marel \& Cioni (2001). A statistical analysis of the distances obtained by including the quoted corrections does not change the results reported above about the LMC distance.

As stated in the previous section, the $\chi^{2}$ values exhibit a large scatter (see Table 1) indicating that the more complex light curve shapes are modeled with lower accuracy and larger residuals. Therefore, we decided also to weigh the best parameters using the $\chi^{2}$ values to define the weights (wts $=1 / \chi^{2}$ ) in order to favour those models that better describe the observed light curve shapes. The resulting weighted distance modulus is equal to $\mu_{0}^{\mathrm{wt}}{ }^{2}=18.56 \mathrm{mag}$ with a standard deviation $\sigma^{\mathrm{wt}} \chi^{2}=0.13 \mathrm{mag}$. We assume that this value is our best estimate of the LMC distance. Using the same $\chi^{2}$-weighted statistics for the excess, we obtain a mean value equal to $E(B-V)^{\mathrm{wt}} \chi^{2}=0.15$ mag with a standard deviation equal to $\sigma^{\mathrm{wt}} \chi^{2}=0.05 \mathrm{mag}$.

The quoted errors represent only the statistical uncertainities, while the systematic is difficult to estimate but depends on the physical and numerical assumptions of the current model sets as well as on residual uncertainties of the

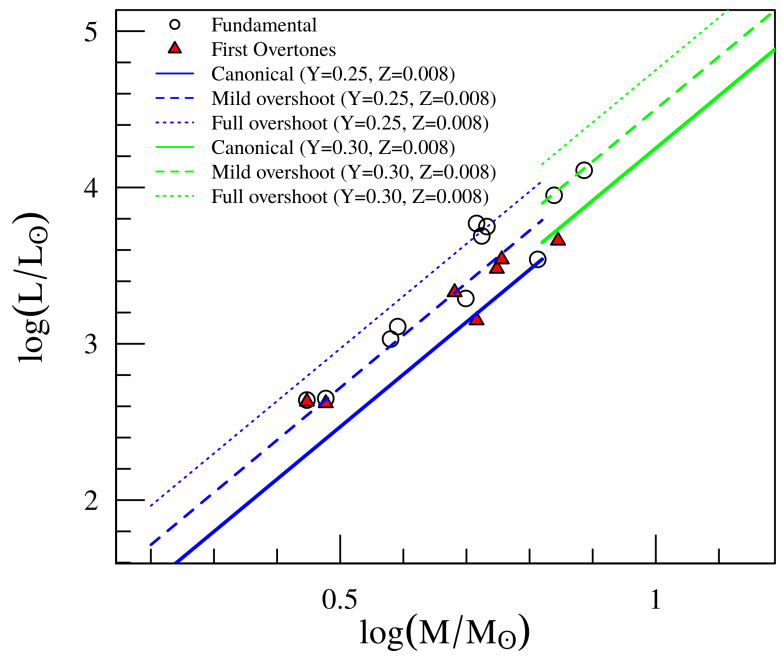

Figure 6. Predicted MLR based on the model fitting results for both F (empty circles) and FO CCs (red filled triangles). The best fitting model location in the MLR plane is compared with an evolutionary MLR obtained by neglecting mass loss, core overshooting and rotation (labelled "Canonical") and with the relations obtained by assuming mild or full overshooting (see text for detail).

adopted atmosphere models. Moreover, the above results for the distance modulus do not take into account projection effects, related to the fact that CCs are not located at the centre of the LMC.

\section{RESULTS}

In this section we use the results obtained for the intrinsic stellar parameters of the investigated CCs to determine constraints both on the predicted MLR and PR relations as well as on the PL and PW relations, at least for the assumed elemental composition. 

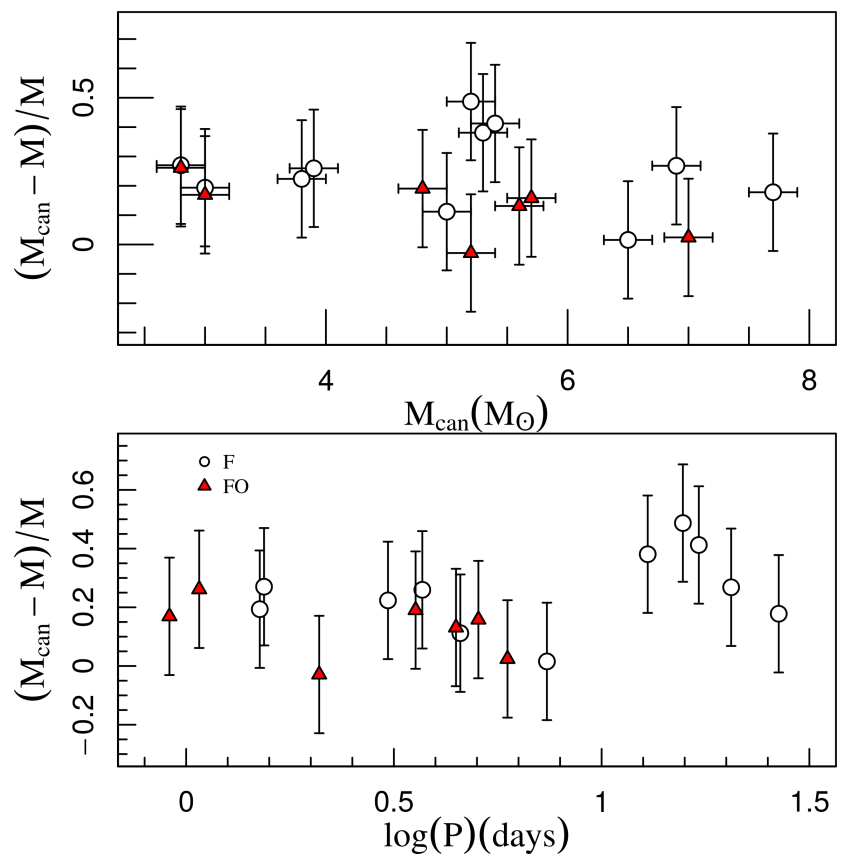

Figure 7. Deviation of the best fitting stellar mass from the value corresponding to the canonical mass for both $\mathrm{F}$ (empty circles) and FO (red triangles) CCs. 


\subsection{The Mass-Luminosity Relation}

Figure 6 displays the MLR for the investigated CCs whose intrinsic stellar parameters were derived from the best fitting models listed in Table 1. These data are compared with the predicted canonical (no overshooting, no mass loss) MLR (Bono et al. 2000) (solid lines) and with the relations obtained by increasing the zero point of the canonical MLR by 0.25 dex (dashed lines) and 0.5 dex (dotted lines) to reproduce the effect of mild and full overshooting ${ }^{5}$, respectively (see Chiosi et al. 1993; Bono, Caputo, Castellani \& Marconi 1999, for details). Inclusion of mass loss and/or rotation would produce a similar increase in the Cepheid luminosity level at fixed mass (see Neilson et al. 2012, for details). As the light curves of $O G L E \_C E P \_L M C \_2019$ are best reproduced adopting a different value of the helium content (see above), in Fig. 6 we also show the MLR for $Y=0.30$, $Z=0.008$ (green lines). Note that this relation is slightly more luminous than those calculated for the standard LMC elemental composition ( $Y=0.25, Z=0.008$ ). According to the location of the variables in the ML plane, the canonical MLR is not strictly satisfied, as the points are spread between the canonical and full overshooting predictions. Even if at this stage we cannot disentangle the role of overshooting, mass loss and rotation in producing the quoted excess luminosity, at fixed mass, the detected dispersion might indicate a combination of these different noncanonical phenomena. Indeed, if only overshooting were efficient, one would in principle expect the same amount of excess luminosity for all stellar masses (within small uncertainties). Rotation produces similar effects as overshooting because it implies a larger He burning core and a brighter luminosity at fixed mass (see e.g. Anderson et al. 2016) On the other hand, if the mass loss process were efficient, this could be inferred from the predicted deviation of the best fitting stellar mass from the value corresponding to the canonical MLR. Such a deviation is represented in Fig. 7 as a function of the canonical mass (top) and of the pulsation period (bottom) for the $\mathrm{CCs}$ in our sample. We note that the expected mass differences range from $0 \%$ to almost $\sim 50 \%$ and are not clearly correlated with the pulsation period or the stellar mass.

\subsection{The Period-Mass-Radius and the Period-Radius relations}

Once we obtain the mass and the radius from the output of the non linear hydrodynamical code, we are able to correlate them with the pulsational period for each $\mathrm{CC}$ to investigate the PMR relation. Assuming the linearized equation introduced above, we obtain:

$$
\begin{array}{r}
\log P=(-1.618 \pm 0.007)+(-0.68 \pm 0.02) \log \left(M / M_{\odot}\right)+ \\
(1.72 \pm 0.01) \log \left(R / R_{\odot}\right)
\end{array}
$$

with a $\sigma=0.005$ dex. The values of the fitted parameters are in excellent agreement with those expected from linear theory (see e.g. Fricke et al. 1972).

If we neglect the mass dependence in the PMR relation,

${ }^{5}$ Corresponding to an extension of the extra-mixing region beyond the Schwarzschild border of about $0.2 H_{p}$ where $H_{p}$ is the pressure scale height (Chiosi et al. 1993).

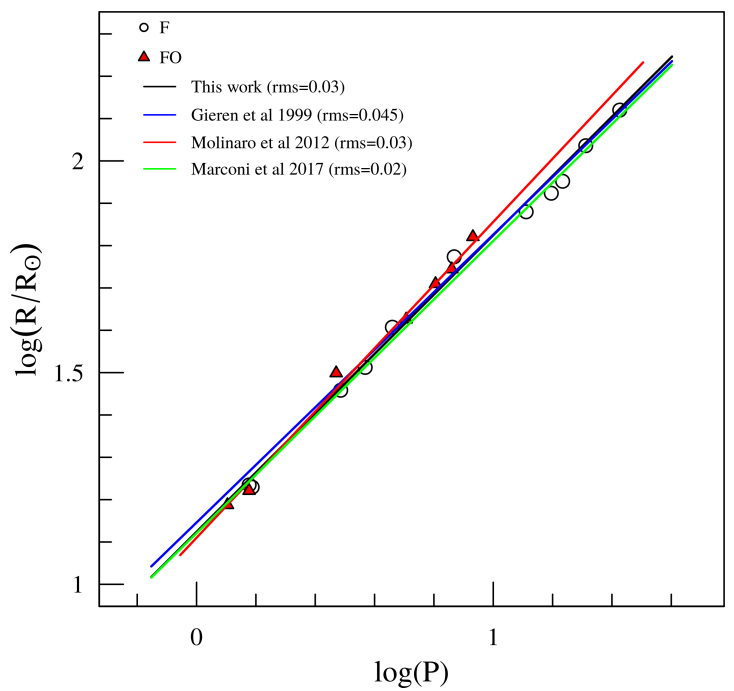

Figure 8. The Cepheid PR relation obtained for the selected sample of 18 sources. The CCs are plotted using empty circles and filled triangles, respectively for Fs and FOs; the FO periods have been fundamentalized (see text). The black line is the result of a linear regression fit to the data (see text). For comparison the PR relations of Gieren et al. (1999) (blue line), Molinaro et al. (2012) (red ine) and Marconi et al. (2017) (green line) are also plotted.

we can obtain the PR relation which is also well studied in the literature (see e.g. Gieren et al. 1999; Molinaro et al. 2011, 2012, and references therein). The location of the 18 investigated CCs in the (PR) diagram is shown in Fig. 8 where Fs are plotted using empty circles and FOs with filled triangles; the periods of the FO pulsators have been fundamentalized using the equation given by Feast, \& Catchpole (1997). A linear regression fit to the data gives us the following PR relation:

$\log \left(R / R_{\odot}\right)=(0.70 \pm 0.02) \log (P)+(1.12 \pm 0.01) ;$

that is displayed with a black line in Fig. 8 with a $\sigma=0.03$ dex. The same figure shows, for comparison, the PR relations of Molinaro et al. (2012) (red line) and Gieren et al. (1999), (blue line), based on two different realizations of the Baade-Wesselink method, and the relation of Marconi et al. (2017) (green line), which was obtained using the model fitting technique for a sample of CCs in the SMC. The PR relation of Molinaro et al. (2012) is based on a sample of 11 CCs belonging to the young LMC blue populous cluster NGC 1866 and 26 Galactic CCs (see also Molinaro et al. 2011), while the PR relation of Gieren et al. (1999) has been derived from a sample of both Galactic and Magellanic CCs. nspection of Fig. 8 and a comparison of the coefficients of the plotted relations reveal that the PR found in this work is in agreement with those of Gieren et al. (1999) and Molinaro et al. (2012). In particular, the result of Gieren et al. (1999) predicts a shallower relation but it is in excellent agreement $(1 \sigma)$ with our PR, while the slope of Molinaro et al. (2012) is steeper than that obtained in this work, though it is consistent within $\sim 2 \sigma$. Concerning the intercepts of relations, they are in excellent agreement $(1 \sigma)$ with that found in the current work. The Baade-Wesselink technique is known to be dependent on the adopted value of the projection factor ( $\mathrm{p}$-factor), which allows to convert 
Table 2. Coefficients of the inferred Period-Radius, PeriodLuminosity and Period-Wesenheit relations (in the $V, I, K_{\mathrm{S}}$ bands), respectively. Columns 4 to 8 represent the slope $(\alpha)$ and the intecept $(\beta)$ with their associated errors $\left(\sigma_{\alpha}, \sigma_{\beta}\right)$, and the rms of the residuals around the fitted relation.

\begin{tabular}{cccccccc}
\hline & Mode & band & $\alpha$ & $\sigma_{\alpha}$ & $\beta$ & $\sigma_{\beta}$ & $\mathrm{rms}$ \\
\hline $\mathrm{PR}$ & & & 0.70 & 0.02 & 1.12 & 0.01 & 0.03 \\
\hline $\mathrm{PL}$ & $\mathrm{F}$ & $V$ & -2.63 & 0.11 & -1.54 & 0.11 & 0.16 \\
& $\mathrm{FO}$ & & -3.10 & 0.16 & -1.95 & 0.09 & 0.13 \\
& $\mathrm{~F}$ & $I$ & -2.93 & 0.11 & -1.95 & 0.10 & 0.15 \\
& $\mathrm{FO}$ & & -3.38 & 0.15 & -2.38 & 0.08 & 0.12 \\
& $\mathrm{~F}$ & $K_{s}$ & -3.30 & 0.09 & -2.43 & 0.09 & 0.13 \\
& $\mathrm{FO}$ & & -3.70 & 0.14 & -2.90 & 0.07 & 0.11 \\
\hline $\mathrm{PW}$ & $\mathrm{F}$ & $W(V, I)$ & -3.39 & 0.10 & -2.58 & 0.09 & 0.14 \\
& $\mathrm{FO}$ & & -3.83 & 0.13 & -3.06 & 0.07 & 0.11 \\
& $\mathrm{~F}$ & $W\left(V, K_{s}\right)$ & -3.39 & 0.09 & -2.55 & 0.09 & 0.13 \\
& $\mathrm{FO}$ & & -3.78 & 0.13 & -3.02 & 0.07 & 0.11 \\
\hline & & & & & & &
\end{tabular}

spectroscopically measured radial velocity into pulsational velocity (see Gieren et al. 1999; Molinaro et al. 2011, 2012; Gallenne et al. 2017; Kervella et al. 2017; Nardetto et al. 2017 , and their references for a discussion). Since the radii obtained from pulsating models are not dependent on this key parameter, comparing the results from the two techniques allows us to put constraints on the $\mathrm{p}$-factor. As for the cases discussed in this work, Molinaro et al. (2012) adopted a constant p-factor of 1.27 , while Gieren et al. (1999) used a period dependent value $(p=1.39-0.03 \log P$ ) from Hindsley, \& Bell (1986). Since both results are consistent with the PR obtained from pulsational models, we are not able to single out one of the two choices as better.

In order to compare the PR relations by Gieren et al. and Molinaro et al., using the same projection factor, we rescaled first the results from the former work to the constant $\mathrm{p}$-factor value adopted by Molinaro et al., and then the results from the latter work to the variable $\mathrm{p}$-factor used by Gieren et al. From this procedure we can conclude that the best agreement with the PR relation in eq. 3 is obtained by using the visual surface brightness technique from Gieren et al., but adopting a constant p-factor as in Molinaro et. al. In particular, in the quoted case we obtain the fitted PR relation $\log \left(R / R_{\odot}\right)=(0.699 \pm 0.017) \log P+(1.11 \pm 0.02)$, which is almost the same as that of eq.3

We also compare our PR relation with that of Marconi et al. (2017), obtained for a sample of SMC CCs using the model fitting technique. Their fitted relation is given by $\log \left(R / R_{\odot}\right)=(0.690 \pm 0.017) \log P+(1.121 \pm 0.016)$ and is fully consistent with our result, indicating that samples with different elemental compositions obey the same PR relation, in agreement with the theoretical results obtained by Bono et al. (1998).

\subsection{The PL Relation}

The mean absolute magnitudes of the best fitting models can be correlated with the corresponding periods to build multi-filter PL relations. In Fig. 9 we show the location of both $\mathrm{F}$ (empty circles) and FO (red filled triangles) best fitting models in the $V, I$ and $K_{\mathrm{s}}$ bands versus period. In

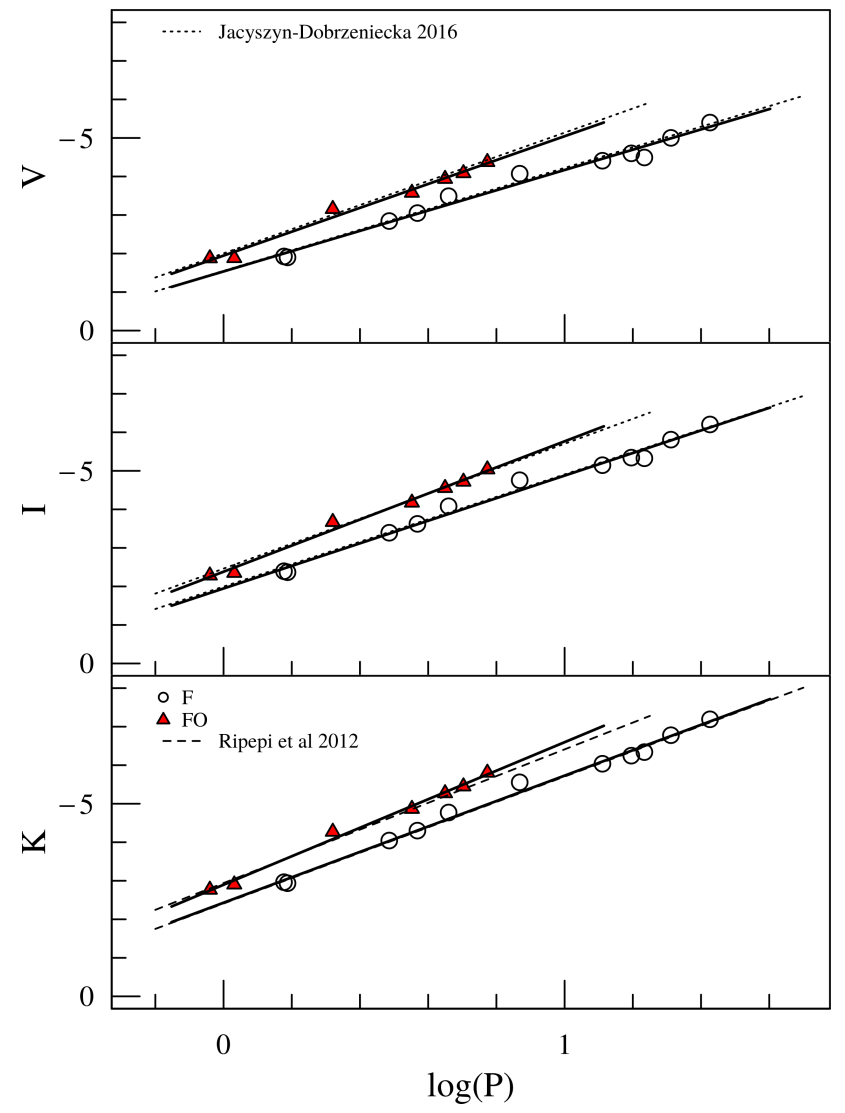

Figure 9. Predicted PL relation in the $V$ (top), $I$ (middle) and $K_{s}$ (bottom) bands based on the model fitting results for both $\mathrm{F}$ (empty circles) and FO (red filled triangles) CCs. The three panels show also PL relations from the literature obtained, namely, by Ripepi et al. (2012b) in the $K_{s}$ band (dashed line) and by Jacyszyn-Dobrzeniecka et al. (2016) in the $V$ and the $I$ bands (dotted lines).

each panel the solid line shows the linear regression fit to the data points whose coefficients are listed in Table 2. To compare our theoretical relations with those obtained by other authors, we have also plotted in the three panels the PL of Jacyszyn-Dobrzeniecka et al. (2016), in the $V$ and $I$ bands, and of Ripepi et al. (2012b) for the $K_{s}$ band. Since the relations given by Jacyszyn-Dobrzeniecka et al. (2016) contain apparent magnitudes, we have first corrected them for absorption using the mean $E(B-V)$ value obtained in this work, and then shifted them using our best estimate of the LMC distance modulus. The comparison of the slope values provided by these authors $\left(\alpha_{V}^{F}=-2.672 \pm 0.006\right.$, $\alpha_{V}^{F O}=-3.133 \pm 0.006, \alpha_{I}^{F}=-2.911 \pm 0.006$ and $\alpha_{I}^{F O}=$ $-3.240 \pm 0.006)$ shows an excellent agreement in the $V$ band. For the $I$ band the two slopes are different, but still consistent thanks to our large error. The $K_{s}$ band PL relations for Fs and FOs of Ripepi et al. (2012b) contain absolute magnitudes and consequently can be directly compared with our results in the corresponding band. From this comparison we found that the coefficients of their relation $\left(\alpha_{K}^{F}=-3.295 \pm 0.018, \beta_{K}^{F}=-2.41 \pm 0.03\right)$ are in excellent agreement with our results for $\mathrm{F}$ pulsators. As for the FO mode their zero-point $\left(\beta_{K}^{F}=-2.94 \pm 0.07\right)$ is in excellent agreement with our value while their slope 


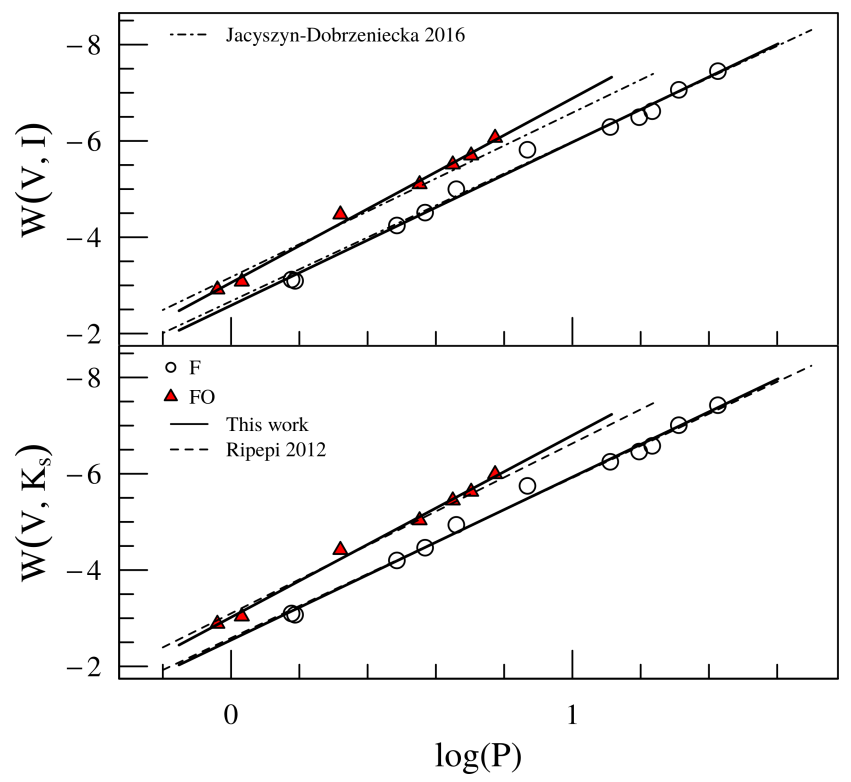

Figure 10. Predicted $\mathrm{PW}$ relations in the $V, I$ (top) and $V, K_{s}$ (bottom) filters for both F and FO pulsators. Symbols are as in the Fig. 9. For comparison with literature results, in the top panel we show also the relation of Jacyszyn-Dobrzeniecka et al. (2016) (dot-dashed lines), while in the bottom panel we plot the relations of Ripepi et al. (2012b) (dashed lines).

$\left(\alpha_{K}^{F}=-3.471 \pm 0.035\right)$ is consistent with our result within $\sim 1.5 \sigma$.

Inspection of the results in Table 2 also suggests that the derived rms of residuals around the PL relations are of the same order of magnitude as that obtained for the obseravtional relations (Ripepi et al. 2012b; Jacyszyn-Dobrzeniecka et al. 2016).

\subsection{The Wesenheit relation}

Finally, it is interesting to compare the PW relations found in the present work with those adopted in the literature (e.g Ripepi et al. 2012b; Jacyszyn-Dobrzeniecka et al. 2016). To estimate the Wesenheit magnitudes for the optical and NIR data, we use the following definitions: $W(V, I)=I-1.55 \times$ $(V-I)$ and $W\left(V, K_{\mathrm{s}}\right)=K_{\mathrm{s}}-0.13 \times\left(V-K_{\mathrm{s}}\right)$, according to recent prescriptions in the literature (see e.g. Soszyński et al. 2015; Ripepi et al. 2016, and references therein). The PW relations for the investigated CCs are shown in Fig. 10, where the symbols have the same meaning as in Fig. 9. The bottom panel shows the relation obtained by combining optical and NIR bands, and the top panel shows the same relation obtained by using only optical bands. The linear regression fits to the data are also shown (solid lines) and the coefficients are reported in Table 2.

The PW equations in the optical bands are provided by Jacyszyn-Dobrzeniecka et al. (2016) and are based on apparent magnitudes. In order to compare them with the results obtained in this work, we have shifted their F and FO relations using our best estimate of the LMC distance modulus $\mu=18.56$ mag. Looking at the top panel of Fig. 10, we note that the relations for $\mathrm{F}$ Cepheids are in excellent agreement, being almost coincident, while the theoretical FO PW relation seems to be steeper than the relation by Jacyszyn-Dobrzeniecka et al. (2016). Indeed, their slope $\alpha_{W(V, I)}^{F O}=-3.414 \pm 0.007$ differs from our estimate by more than $3 \sigma$.

The $W\left(V, K_{s}\right)$ relations provided by Ripepi et al. (2012b) are expressed using absolute magnitudes and consequently can be directly compared with our results. Their equation for $\mathrm{F}$ pulsators $\left(W\left(V, K_{s}\right)=(-3.325 \pm\right.$ $0.014) \log P+(-2.59 \pm 0.03))$ is in excellent agreement with our result (see Table 2), with both slope and intercept being consistent within less than $1 \sigma$. The coefficients of their FO equation $\left(W\left(V, K_{s}\right)=(-3.530 \pm 0.025) \log P+(-3.10 \pm\right.$ $0.07)$ ), are consistent with our estimates within $\sim 1.5 \sigma$.

\section{CONCLUSIONS}

We considered a sample of $11 \mathrm{~F}$ and $7 \mathrm{FO}$ CCs in the LMC with optical photometry from the OGLE III database and NIR photometry from the VMC survey. By assuming first approximation elemental composition typical of LMC CCs ( $Y=0.25, Z=0.008$ ), for each selected pulsator, we built isoperiodic model sequences varying the intrinsic stellar properties (effective temperature, mass/luminosity) in order to match the period, and the shape of the observed light curves in the $V, I$ and $K_{\mathrm{s}}$ bands. The resulting models directly provide information on the mass, the effective temperature, the luminosity and in turn the individual distance of each selected target VMC CC. On this basis we obtained the following results:

- From the inferred apparent distance moduli, adopting the extinction law by Cardelli et al. (1989) we obtained an estimate of the intrinsic distance modulus for every star in our sample. We decided to weigh these values using the best fitting $\chi^{2}$ to give and an estimate of the LMC distance modulus. Our procedure provides a value of $\mu_{0}=18.56 \mathrm{mag}$ with a standard deviation of $0.13 \mathrm{mag}$, in agreement with the most recent literature values (Marengo et al. 2010; Marconi \& Clementini 2005; Ripepi et al. 2012a; Pietrzyński et al. 2013; de Grijs et al. 2014; Jacyszyn-Dobrzeniecka et al. 2016). We note that our best value for the LMC distance modulus is in perfect agreement with the results of one of our previous applications ( $18.53 \pm 0.05 \mathrm{mag}$ Bono et al. 2002) and the estimate by Keller et al. (2006), $18.54 \pm 0.018 \mathrm{mag}$, obtained using a similar approach for a sample of bump Cepheids covering a pulsation period range centred on 10 days, an almost complementary range compared to the sample analyzed in this work.

- Considering the geometric correction according to the model by van der Marel \& Cioni (2001) in the estimation of the LMC distance has no effect on the quoted results.

- the MLR is clearly more luminous than the evolutionary MLR that neglects overshooting, mass loss and rotation, thus suggesting a high efficiency of at least one of these noncanonical phenomena.

- A PR relation in agreement with the literature results, in particular with the relation of Gieren et al. (1999).

- Theoretical PL relations in the $V, I$ and $K_{\mathrm{s}}$ bands adequately reproduce the observed intrinsic scatter of the PL distribution.

- Theoretical PW relations are in agreement with the 
empirical LMC Wesenheit relations recently presented by Ripepi et al. (2012b).

We note that for one long-period Cepheid we needed to vary the elemental composition in order to obtain a satisfactory fit. In particular for CC OGLE_CEP_LMC_2019 an enhanced helium abundance $Y=0.30$ was required in order to fit the observed curve. The possible presence of a fraction of helium enriched CCs has been recently theoretically investigated by Carini et al. (2017), following previous indications of the presence of multiple stellar populations in young LMC star clusters (see e.g. Milone et al. 2016, and references therein).

In the future we also plan to extend the application to other samples of pulsators in order to better constrain their PL and PW relations and to test the accuracy of the method through application to the light curves of Galactic CCs with Gaia parallaxes (Gaia Collaboration et al. 2018). The latter comparison, once we have fixed the distance to the Gaia results, will also allow us to put strong constraints on the predicted stellar masses, the MLR, and, once the metallicity is precisely constrained by complementary spectroscopic data, the helium to metal enrichment ratio.

\section{ACKNOWLEDGEMENTS}

The authors are grateful to the referee Prof. Jan Lub for the very constructive comments that significantly improved the paper.

This work is based on observations collected at the European Organisation for Astronomical Research in the Southern Hemisphere under ESO programme 179.B-2003. We thank the CASU and the WFAU for providing calibrated data products under support of the Science and Technology Facility Council (STFC) in the UK. M-R.C. acknowledges funding from the European Research Council (ERC) under the European Union's Horizon 2020 research and innovation programme (grant agreement No 682115).

\section{References}

Anderson R.I. et al., 2016, A\&A, 591, 28

Anderson, R. I., Ekström, S., Georgy, C., et al. 2017, European Physical Journal Web of Conferences, 06002

Bono, G., Caputo, F., \& Marconi, M. 1998, ApJ, 497, L43

Bono G., Caputo F., Castellani V., Marconi M., 1999, ApJ, 512, 711

Bono G., Marconi M., Stellingwerf R. F., 1999, ApJS, 122, 167

Bono, G., Gieren, W. P., Marconi, M., et al. 2001, ApJ, 563, 319

Bono, G., Caputo, F., Cassisi, S, Marconi, M., Piersanti, L., Amedeo, T., 2000, ApJ, 543, 955.

Bono, G., Castellani, V., \& Marconi, M. 2002, ApJL, 565, L83

Caputo F., Marconi, M., Musella, I., 2000, A\&A, 354, 610

Caputo, F. , Bono, G., Fiorentino, G., Marconi, M., Musella, I. , 2005, ApJ, 629, 1021-1033

Cardelli, J.A., Clayton, G.C., Mathis, J.S., 1989, ApJ, 135, 5-10

Carini, R., Brocato, E., Raimondo, G., Marconi, M., 2017, MNRAS, 469, 1532

Chiosi, C., Wood, P. R., \& Capitanio, N. 1993, ApJS, 86, 541

Christy, R. F. 1970, JRASC, 64, 8

Cioni, M.R.L. , 2011, A\&A, 527, 22

Cross N. J. G., Collins R. S., Mann R. G., et al. 2012,A\&A , 548, A119
Dalton, G. B., Caldwell, M., Ward, A. K., et al. 2006, SPIE, 62690X

de Grijs, R., Wicker, J. E., \& Bono, G. 2014, AJ, 147, 122

Eddington, A. S. 1926, The Internal Constitution of the Stars (Cambridge: Cambridge Univ. Press)

Emerson J., McPherson A., Sutherland W. ,2006, The Messenger, $126,41-42$

Feast, M. W., \& Catchpole, R. M. 1997, MNRAS, 286, L1

Freedman, W. L., Wilson, C. D., Madore, B. F., 1991, ApJS, 24, 14

Fricke, K.; Stobie, R. S.; Strittmatter, P. A., 1971, ApJ, 171, 593

Gaia Collaboration, Brown, A. G. A., Vallenari, A., et al. 2018, A\&A, 616, A1

Gallenne, A., Kervella, P., Mérand, A., et al. 2017, A\&A, 608, A18

Gieren, W.P., Moffett, T.J., Varnes, T.G.,1999, ApJ, 512, 553-557

González-Fernández, C., Hodgkin, S. T., Irwin, M. J., et al. 2018, MNRAS, 474, 5459

Hindsley, R., \& Bell, R. A. 1986, PASP, 98, 881

Jacyszyn-Dobrzeniecka, A. M., Skowron, D. M., Mróz, P., et al. 2016, ACTAA, 66, 149

Keller S.C., Wood P.R. , 2006, ApJ, 642, 834-841

Kervella, P., Trahin, B., Bond, H. E., et al. 2017, A\&A, 600, A127

Luck, R. Earle, Moffett, Thomas J., Barnes, Thomas G., III, Gieren, Wolfgang P. 1998, AJ, 115, 605

Madore B.F., 1982, ApJ, 253, 575-579

Marconi, M., \& Clementini, G. 2005, AJ, 129, 2257

Marconi, M., Molinaro, R., Bono, G, et al. ,2013, ApJ, 768, L6

Marconi, M., Molinaro, R., Ripepi, V., et al. ,2017, MNRAS, 466, 3206-3016

Marengo, M., Evans, N. R., Barmby, P., et al., 2010, ApJ, 725, $2392-2400$

Milone, A. P., Marino, A. F., D'Antona, F., Bedin, L. R., Da Costa, G. S., Jerjen, H.; Mackey, A. D. 2016, MNRAS, 458, 4368

Molinaro, R., Ripepi, V., Marconi, M., Musella, I., Brocato, E., Mucciarelli, A., Stetson, P. B., Storm, J., Walker, A. R., 2012, ApJ, 748, 69

Molinaro R., Ripepi V., Marconi M., Bono G., Lub J., Pedicelli S., Pel J.W., 2011, MNRAS, 413, 942

Moretti, M. I., Clementini, G., Muraveva, T., et al. ,2014, MNRAS, 437, 2702-2719

Moretti, M. I., Clementini, G., Ripepi, V., et al., 2016, MNRAS, 459, 2, 1687-1697

Muraveva, T.; Palmer, M.; Clementini, G.; Luri, X., Cioni, M.-R. L., Moretti, M. I., Marconi, M., Ripepi, V., Rubele, S, 2015, ApJ, 807, 127

Muraveva, T., Subramanian, S., Clementini, G., et al. 2018, MNRAS, 473, 3131

Nardetto, N., Poretti, E., Rainer, M., et al. 2017, A\&A, 597, A73

Neilson, H.R., Langer, N., Engle, S.G., Guinan, E., Izzard, R., 2012, ApJ, 760L, 18

Pietrzyński, G., Graczyk, D., Gieren, W., et al. 2013, Nature, 495, 76

Ripepi, V., Moretti, M. I., Clementini, G., et al. 2012, APSS, 341, 51

Ripepi, V., Moretti, M. I., Marconi, M., et al. 2012, MNRAS, 424, 1807

Ripepi, V., Marconi, M., Moretti, M. I., et al. ,2014, MNRAS, $437,2307-2319$

Ripepi, V., Moretti, M. I., Marconi, M, et al. ,2015, MNRAS, 446, 3034-3061

Ripepi, V., Marconi, M., Moretti, M. I., et al. ,2016, ApJS, 224, 21

Ripepi, V., Cioni, M.-R. L., Moretti, M. I., et al. ,2017, MNRAS, $472,808-827$

Romaniello, M., Primas, F., Mottini, M., et al. 2008, A\&A, 488, 731 


\section{Fabio Ragosta et al.}

Soszyński, I., Udalski, A., Szymański, M. K., et al. 2010, ACTAA, 60,165

Soszyński, I., Udalski, A., Szymański, M. K., et al. 2015, ACTAA, 65,297

Stobie R.S., 1969, MNRAS, 144, 511

van der Marel, R. P., \& Cioni, M.-R. L. 2001, AJ, 122, 1807 